\title{
Optical properties of nematic liquid crystals doped with gold nanorods
}

\author{
S. Torgova ${ }^{1}$, E. Pozhidaev ${ }^{1}$, A. Lobanov ${ }^{1}$, M. Minchenko ${ }^{1}$ and B. Khlebtsov ${ }^{2}$ \\ ${ }^{I}$ P.N. Lebedev Physical Institute, Russian Academy of Sciences, \\ Leninskii prospect, 53, Moscow 119991, Russia; \\ ${ }^{2}$ Institute of Biochemistry and Physiology of Plants and Microorganisms, \\ Russian Academy of Sciences, prospect Entuziastov, 13, Saratov 410049, Russia; \\ Phone: +7 (499) $1326459^{1}$, +7 (499) $1326116^{1}$; \\ E-mail: sofia.torgova@mail.ru ; epozhidaev@mail.ru'; lobanov@sci.lebedev.rul';mminchenko@mail.ru ; \\ khlebtsov@ibppm.sgu.ru²
}

\begin{abstract}
Composites consisting of nematic liquid crystal (5-CB) and gold nanorods have been elaborated and investigated with a polarizing microscope. It was detected that the nanorods form inside the oriented liquid crystal matrix their own self-assembling well-ordered structures. Nanorods ordered structures appear as a result of aligning layers action and provides defects corresponding to the spatial distortion of the nematic liquid crystal director field.
\end{abstract}

Keywords: liquid crystal, gold nanorod, self-assembling structures.

Manuscript received 18.12.09; accepted for publication 25.03.10; published online 30.04.10.

\section{Introduction}

A new direction in studying the nanoparticles is related with their insertion to a liquid crystal matrix as a new non-synthetic method for modification of properties inherent to existing liquid crystals. Today it is a top of scientific interest, and a huge amount of papers are aimed at this subject. Here, we mention just some of them, where investigated nanoparticles are carbon nanotubes, metallic spheres and ferroelectric nanoparticles [1-7]. In this paper, new results concerning the introduction of gold nanorods into nematic liquidcrystal matrixes are reported.

\section{Experiment}

In our experiments, we used hydrosols of gold nanorods that were synthesized according to the methods presented in $[8,9]$. The nanorods possess the shape of hemispherical ended cylinders. Average nanoparticle sizes were as follows: length about $70-100 \mathrm{~nm}$ and thickness about $15-20 \mathrm{~nm}$. The concentration of particles in hydrosols was not less than $10^{11} \mathrm{ml}^{-1}$. There were used nanorods with surfactant CTAB (Cetyl trimethyl ammonium bromide) dispersed in water.
The gold nanorods were inserted into liquid-crystal (LC) matrixes in very small amount (0.02 vol.\%).

In order to obtain the composites based on gold nanorods and nematic LC, the latter was dissolved in ethanol, after this, water suspension of nanoparticles covered by surfactant was added, and then the solvents were removed in vacuum. The obtained nanocomposites (NC) were treated by ultrasound before the insertion into the cell. The optical properties of NC were observed by a polarization microscope. The results obtained are shown in Figs 1 to 3.

\section{Results and discussion}

We investigated an influence of the insertion of very small percentage of gold nanorods into LC matrix. We observed that gold nanorods form inside the ordered structure of liquid crystal different self-assembling wellordered structures (defects) corresponding to spatial distortion of the LC director field. The size of these defects is much larger than that of the particles themselves and varies from few to hundred micrometers. It is necessary to underline that the size, shape, orientation and ordering of the defects depend on many reasons, namely: the technology of cell surface 
treatment, the thickness of $\mathrm{NC}$ layer, the cell filling conditions, the nature of LC matrix. The most interesting result is presented in Fig. 1. It has been shown that orientation of the LC principal optical axis (LC director) and long axis of the self-assembling ordered nanorod structures are matched together. On the other hand, the LC director and the principal optical axis of defects never match together, which can be explained by the difference between surface tensions of LC and of gold nanorods covered by surfactant: the surfactant surface tension is considerably less than that of liquid crystals. The appearance of some defects caused by insertion of nanoparticles into LC matrix under permanent electric field was mentioned in [6].

The most ordered defects were observed in the cells treated for planar alignment, where the LC layer thickness is $1.8 \mu \mathrm{m}$. Asymmetric boundary conditions were used for manufacturing the cells. In this approach, only one indium tin oxide (ITO) surface of LC cells was covered with the polyimide (PMDA-ODA) possessing the structure of repeated pyromellitic dianhydriden (PMDA) and 4,4'-oxidianiline (ODA) units, while another one was simply washed in $N, N$-dimethyl formamide (DMF) and covered with $1.8 \mu \mathrm{m}$ calibrated spacers.

The concentration of nanorods in nanocomposite is $0.02 \%$, and insertion of NC in the cell was done at the temperature higher than transition of $\mathrm{LC}$ from the nematic to isotropic phase. Under these conditions, the defects have elongated stick-like shape, forming wellordered regular planar structures, which are perpendicular to the LC director (see Fig. 1).

It is possible to understand the importance of the boundary conditions influence on formation of defects and their ordering by comparison of Figs 1, 2 and 3 . There is no any preferable direction of the principal optical axis of planar defects in a cell without any aligning layers (Fig. 2), while the liquid crystal itself is aligned homeotropically.

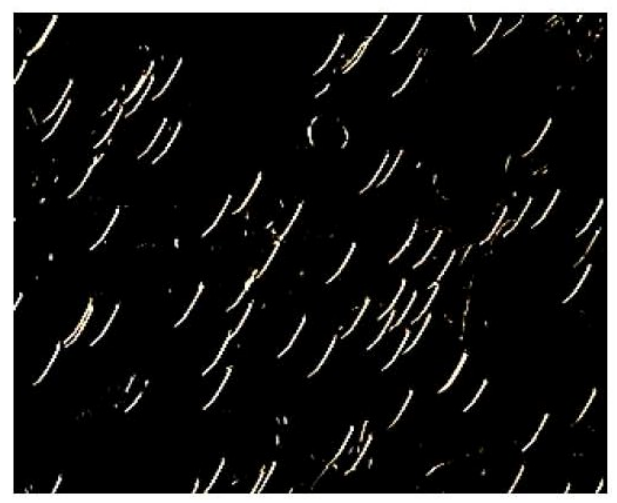

Fig. 1. Microphoto of the NC layer in the cell with the thickness $1.8 \mu \mathrm{m}$ treated for planar alignment of the liquid crystal. The cell was filled using the capillary method at the temperature higher than the clearing point. The photo was done between crossed polarizers at room temperature. The size is $500 \times 400 \mu \mathrm{m}$.

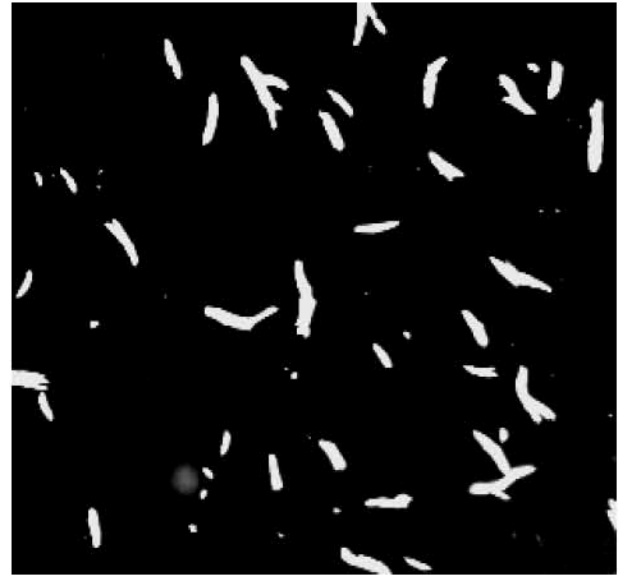

Fig. 2. Microphoto of the NC droplet squeezed between two glasses without any aligning layers, made with crossed polarizers at room temperature. The size is $500 \times 400 \mu \mathrm{m}$.

The same NC inserted into the homeotropically aligned cell under the same conditions shows a completely different result - homeotropic alignment of both LC and defects (see Fig. 3).

The possible explanation of the defects formation is as follows: filling the cell by using the capillary method leads to planar orientation of nanorods and the main part of NLC matrix. Surfactant of the nanorod anchors with the aligning layer of the cell. This surfactant locally orients the NLC matrix perpendicular to the surface of nanorod during the transition from isotropic liquid to nematic phase. This local orientation is schematically shown in Fig. 4. This hypothesis is in a good agreement with the experimental data.

The clearing point of the defects in NC is at least 23 degrees higher than the clearing point of 5-CB itself $\left(35^{\circ} \mathrm{C}\right)$. In Fig. 5 , we present the photos of the cooling process from the isotropic phase.

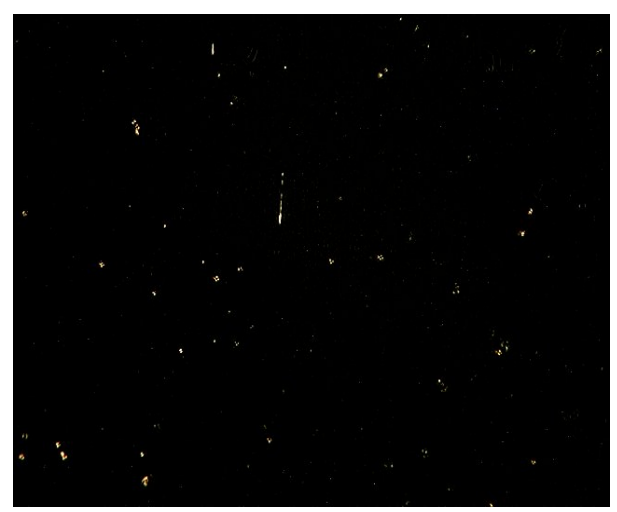

Fig. 3. Microphoto of NC, LC in the cell with the liquid crystal layer thickness $1.8 \mu \mathrm{m}$ treated for homeotropic alignment by chromolane. The cell was filled using the capillary method at the temperature higher than the clearing point. Photo was done between crossed polarizers at room temperature. 


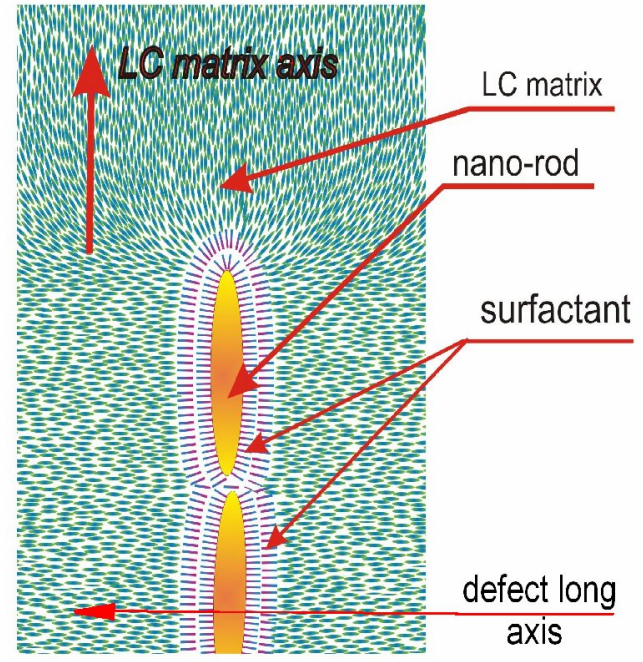

Fig. 4. Schematic explanation of the defect formation.

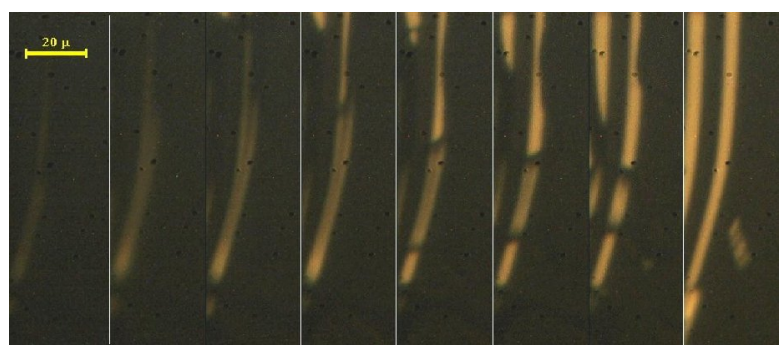

Fig. 5. Microphoto of NC cooling from the isotropic phase.

As seen from Fig. 5, appearance of the defects precedes to the formation of the matrix nematic phase.

The position and size of defects in $\mathrm{NC}$ do not change, after the heating till the clearing point of 5-CB and cooling run from the isotropic phase. We succeeded to "cancel" the self-assembling clusters only at temperatures higher than $75^{\circ} \mathrm{C}$. Probably, it demonstrates that the anchoring of the nanorod surfactant with the aligning layer of the cell is sufficiently more strong that the liquid crystal themselves, but it needs further investigations.

\section{Conclusions}

In this work, nanocomposites consisting of nematic liquid crystals (NLC) and gold nanorods have been obtained. We observed that gold nanorods form inside the oriented liquid crystal matrix different selfassembling well-ordered structures (defects) correspondding to spatial distortion of the NLC director field.

The size, shape, alignment and ordering of the defects depend on many reasons and are very sensitive to any change of the experimental conditions.

The NLC director alignment (coincident with the main optical axis) and the long axis of self-assembling well-ordered structures never match together (in the main cases there are perpendicular to each other), which can be explained due to the difference between the surface tension of LC and of the gold nanorods covered by surfactant, if taking into account the long-range order interaction inside this system.

\section{Acknowledgements}

The work was supported by Russian Foundation of Basic Researches, grant N 08-02-01074a.

\section{References}

1. Y. Shiraishi, K. Maeda, H. Yoshikawa, J. Xu, N. Toshima and S. Kobayashi // Appl. Phys. Lett. 1(15), p. 2845 (2002).

2. Y. Reznikov, O. Buchnev, O. Tereshchenko, V. Reshetnyak, A. Glushchenko and J. West // Appl. Phys. Lett. 82(12), p. 1917 (2003).

3. S. Kobayashi, T. Miyama, N. Nishida, Y. Sakai, H. Shiraki, Y. Shiraishi and N. Toshima // J. Disp. Technol. 2, p. 121 (2006).

4. S. Kundu, M. Akimoto, I. Hirayama, M. Jnoue, S. Kobayashi and K. Takatoh // Jpn. J. Appl. Phys. 47, p. 4751 (2008).

5. L. Scolari, S. Gauza, H. Xianyu, L. Zhai, L. Eskildsen, T.T. Alkeskjold, S.T. Wu and A. Bjarklev // Opt. Express 17, p. 3754 (2009).

6. S.I. Trashkeev, G.N. Grachev and G.A. Posdnjakov // Zhurnal Tekhnich. Fiziki 77(9), p. 85 (2007), in Russian.

7. H. Qi, B. Kinkead and T. Hegmann // Adv. Funct. Mater. 18(2), p. 212-221 (2008).

8. H. Liao and J.H. Hafner // Chem. Mater. 17, p. 4636 (2005).

9. B. Nikoobakht and M.A. E1-Stayed // Chem. Mater. 15, p. 1957 (2003). 